\section{Science-Business eXchange}

\title{
Paclitaxel plants routes in bacteria
}

\section{By Michael J. Haas, Senior Writer}

U.S. and Singaporean researchers have engineered strains of Escherichia coli that produce two precursors of the cancer drug Taxol paclitaxel at up to 100 -fold higher yields than previously attained in microorganisms. ${ }^{1}$ The results suggest that complete synthesis of paclitaxel in bacteria is feasible and provide a shortcut that could help generate new bioactive analogs derived from the precursors taxadiene and taxadien-5 $\alpha$-ol.

Paclitaxel was isolated from the Pacific yew tree (Taxus brevifolia) and is now produced via chemical reaction sequences consisting of 35 or more steps that result in overall paclitaxel yields of less than $1 \%$. There is a shorter reaction sequence that starts with 10-deacetylbaccatin III, a paclitaxel precursor isolated from the European yew tree (T. baccata), but that reaction depends on a plant source for starting material.

The inefficiency of both approaches has driven a continuing search for new ways to produce the drug.

One alternative to chemical synthesis is biosynthesis in microorganisms (see Figure 1, “On the road to paclitaxel”). Such approaches tap the natural capacity of bacteria and yeast to produce isoprenoids-a class of five-carbon molecules that are building blocks for more complex molecules-and involve engineering the microorganism to express plant enzymes that assemble the isoprenoids into desired structures. ${ }^{2-4}$

However, attempts to engineer E. coli and Saccharomyces cerevisiae to produce taxadiene-the core hydrocarbon precursor of paclitaxel-have resulted in yields too low to be useful for subsequent chemical reactions..$^{5,6}$

The U.S.-Singapore team, led by Gregory Stephanopoulos and Blaine Pfeifer, hypothesized that the lackluster taxadiene yields in bacteria and yeast were caused in part by cellular toxicity of intermediates, adverse effects of the vectors used to increase gene expression, and unknown pathways and molecules that inhibited taxadiene production.

To overcome those obstacles, the team performed a combinatorial analysis to probe how different promoter strengths and different copy numbers of genes encoding four bacterial and two plant enzymes affected taxadiene production in a total of 32 engineered strains of E. coli.

The results of the analysis allowed the team to engineer E. coli expressing a specific combination of the six genes that produced taxadiene in yields that were about 100-fold higher than those previously attained in bacteria or yeast.

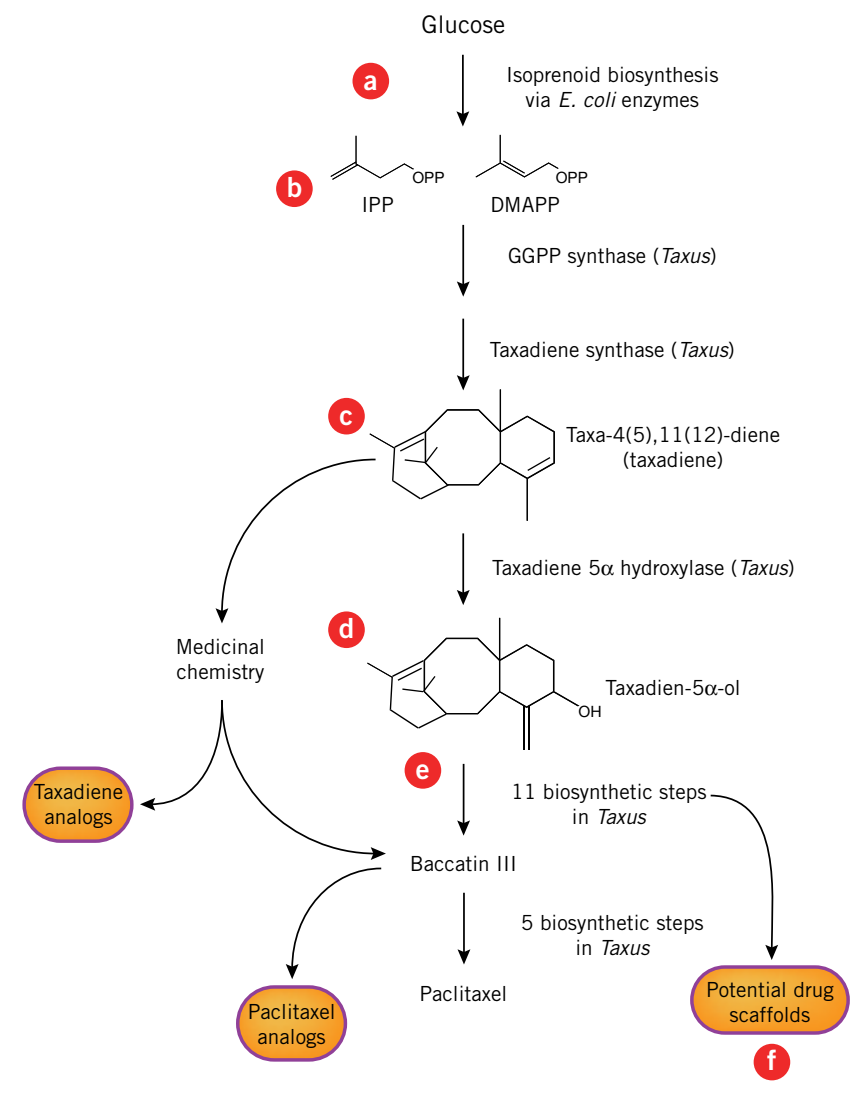

Figure 1. On the road to paclitaxel. Optimized expression of bacterial and plant enzymes in Escherichia coli could produce Taxol paclitaxel intermediates in sufficient yields for subsequent drug discovery research.

As described in Science, [a] E. coli enzymes convert glucose into the isoprenoids [b] isopentenyl pyrophosphate (IPP) and dimethylallyl pyrophosphate (DMAPP), which three Taxus enzymes in turn assemble into [c] the paclitaxel intermediate taxa-4(5),11(12)-diene (taxadiene) and oxidize to [d] taxadien- $5 \alpha$-ol.

Conversion of [c] to [d] is the first of 11 biosynthetic steps to the key paclitaxel precursor [e] baccatin III, a pathway that could also yield [f] new scaffolds for drug discovery research.

Additionally, [c] may be oxidized and converted to precursor [e] baccatin III by medicinal chemistry methods such as hydrocarbon oxidation that might also yield new analogs for drug discovery research.

The incorporation of a third plant enzyme-T. brevifolia cytochrome P450 (p450) - into the bacteria enabled the oxidation of taxadiene into taxadien- $5 \alpha$-ol at yields that could be used for further chemical reactions, Stephanopoulos told SciBX.

Stephanopoulos is a professor of chemical engineering and biotechnology at the Massachusetts Institute of Technology (MIT), and Pfeifer is an assistant professor of chemical and biological engineering at Tufts University. 
The group also included researchers from the Singapore-MIT Alliance (SMA), a collaboration between MIT, the National University of Singapore and Nanyang Technological University.

The data were reported in Science. ${ }^{1}$

"This paper shows how to tap into biosynthetic mechanisms to obtain more of what we get in nature and to produce compounds that are different from what we get in nature," said Paul Wender, professor of chemistry at Stanford University.

"This is a phenomenal advance," said M. Christina White, associate professor of chemistry at the University of Illinois at Urbana-Champaign (UIUC). Having access to large quantities of taxadiene "would significantly advance our program in developing an efficient synthesis of Taxol and derivatives via a hydrocarbon oxidation route."

White's group is interested in carrying out the total synthesis of paclitaxel, starting with a hydrocarbon skeleton and introducing oxidation at predictable sites via an iron-based catalytic method. ${ }^{7}$ She told SciBX that the best current method for synthesizing taxadiene ${ }^{8}$ is unlikely to provide her group with sufficient material to optimize the synthesis of paclitaxel intermediates.

With enough taxadiene on hand "we certainly would anticipate being able to oxidize both secondary and tertiary carbon sites on the taxadiene skeleton that would lead to baccatin III," which is just a few reaction steps away from paclitaxel, White said.

She added that oxidation at other sites on the taxadiene skeleton may also lead to new structures with interesting biological properties.

\section{New ways forward}

Wender said access to taxadiene would aid researchers trying to develop new synthetic routes to paclitaxel but thinks the bigger ramifications "are that the findings could be transferable to other biosynthetic systems."

The result, he said, could be to increase yields of other natural products for which supplies are limited, such as the antimalarial compound artemisinin and its analogs or prostratin and its analogs, which have been shown to activate latent HIV so that it could be targeted by antiretrovirals. ${ }^{9,10}$

Sarah O'Connor, associate professor of chemistry at MIT, agreed that the findings described in Science offer a general method for engineering the production of plant-derived compounds in E. coli.

She noted that large-scale biosynthesis of plant-derived products in bacteria is an attractive prospect because E. coli grows much faster than plant-based systems.

However, O'Connor said, in bacteria that heterologously express plant enzymes, a key impediment has been that compound yields can drop substantially as each step of a plant pathway is incorporated into the bacteria, "particularly with oxidative steps catalyzed by microsomal p450 enzymes, which are found ubiquitously in plant biosynthesis."
The Science study addressed these issues in the early steps of the paclitaxel pathway by "optimizing the upstream portions of the pathway very nicely and providing a really great system for looking at some of the challenging, oxidative downstream steps," said O'Connor.

She added: "As the authors point out in the paper, a number of additional enzymatic steps remain to be added to complete the biosynthesis of Taxol. But this system provides the starting point to explore how a long plant pathway can be effectively reconstituted in E. coli."

Wender thinks the main value of the new method will be developing intermediates that are further along the paclitaxel pathway than taxadiene and taxadien- $5 \alpha$-ol.

Indeed, Stephanopoulos said the next step is to engineer the E. coli system to increase the yield of taxadien-5 $\alpha$-ol and incorporate additional plant enzymes on the paclitaxel pathway downstream of taxadien- $5 \alpha$-ol.

Although the primary goal is the biosynthesis of baccatin III, Stephanopoulos said the team is also interested in studying the molecules produced in those downstream steps as potential drug scaffolds.

Pfeifer said the team also envisions using its E. coli system to produce paclitaxel analogs that could have greater potency and better bioavailability and pharmacokinetics than the parent molecule.

MIT has submitted a patent application covering the findings. Stephanopoulos said the IP is available for licensing or partnering.

Haas, M.J. SciBX 3(40); doi:10.1038/scibx.2010.1199

Published online Oct. 14, 2010

\section{REFERENCES}

1. Ajikumar, P.K. et al. Science; published online Sept. 30, 2010; doi:10.1126/science.1191652

Contact: Gregory Stephanopoulos, Massachusetts Institute of Technology, Cambridge, Mass.

e-mail: gregstep@mit.edu

Contact: Blaine Pfeifer, Tufts University, Medford, Mass. e-mail: blaine.pfeifer@tufts.edu

2. Farmer, W.R. \& Liao, J.C. Nat. Biotechnol. 18, 533-537 (2000)

3. Chang, M.C.Y. \& Keasling, J.D. Nat. Chem. Biol. 12, 674-681 (2006)

4. Morrone, D. et al. Appl. Microbiol. Biotechnol. 85, 1893-1906 (2010)

5. Huang, Q. et al. Bioorg. Med. Chem. 9, 2237-2242 (2001)

6. Engels, B. et al. Metab. Eng. 10, 201-206 (2008)

7. Chen, M.S. \& White, M.C. Science 327, 566-571 (2010)

8. Rubenstein, S.M. \& Williams, R.M. J. Org. Chem. 60, 7215-7223 (1995)

9. Gustafson, K.R. et al. J. Med. Chem. 35, 1978-1986 (1992)

10. Bocklandt, S. et al. Antiviral Res. 59, 89-98 (2003)

\section{COMPANIES AND INSTITUTIONS MENTIONED}

Massachusetts Institute of Technology, Cambridge, Mass.

Nanyang Technological University, Singapore

National University of Singapore, Singapore

Singapore-MIT Alliance, Singapore

Stanford University, Stanford, Calif.

Tufts University, Medford, Mass.

University of IIlinois at Urbana-Champaign, Urbana, III. 